\title{
Atención a la diversidad en las aulas madrileñas. Opiniones de los docentes
}

\author{
Silvia García Sánchez - Universidad Autónoma de Madrid (D) 0000-0002-0144-4389 \\ Marta Garrote Salazar - Universidad Autónoma de Madrid (D) 0000-0002-5566-9073
}

Recepción: 22.01.2021 | Aceptado: 01.02.2021

Correspondencia a través de ORCID: Marta Garrote Salazar

iD 0000-0002-5566-9073

Citar: García Sánchez, S. y Garrote Salazar, M. (2021). Atención a la diversidad en las aulas madrileñas. Opiniones de los docentes. REIDOCREA, 10(1), 1-19.

Resumen: La atención a la diversidad defiende el derecho fundamental a la educación de cada niño y niña diseñando currículos educativos adaptados a las necesidades de todo el alumnado. El presente estudio describe el concepto de atención a la diversidad en la escuela mediante el análisis de las perspectivas docentes. Los participantes $(n=103)$ son docentes de Educación Infantil y Primaria de la Comunidad Autónoma de Madrid. Sus opiniones se recogieron mediante un cuestionario que indaga sobre la atención a la diversidad desde distintas perspectivas. Los resultados reflejan que los y las docentes son conscientes de la importancia de la formación en atención a la diversidad y de sus carencias formativas en esta área. Sin embargo, aseguran conocer herramientas inclusivas y ponerlas en práctica en el aula. Los especialistas en necesidades educativas son el grupo que muestra un perfil más inclusivo. Por otro lado, los datos recogidos indican que el sexo y la edad del profesorado influyen a la hora de atender a la diversidad, mientras que los años de experiencia docente parecen no afectar. Estos resultados sugieren que la formación en atención a la diversidad es clave para garantizar el derecho a la educación de todo el alumnado.

Palabra clave: Educación inclusiva

Attention to diversity in Madrilenian classrooms. Teachers' opinions

Abstract: Attention to diversity stands up for children's rights to education and the design of accessible education curricula for everyone. This paper describes the current situation of attention to diversity at schools through the analysis of teacher's perspectives. The participants $(n=103)$ are Preschool and Primary Education teachers in the Comunidad Autónoma de Madrid. Their opinions were gathered using a questionnaire based on different issues about inclusive education and attention to diversity. The results show that the sample agreed on the importance of training in attention to diversity and they recognised a shortage of it due to several reasons. However, they affirmed that they know inclusive tools which they put into practice in their classrooms. Those teachers specialised in education needs are the ones who display a more inclusive profile. Furthermore, data show that teachers' sex and age influence their conception of attention to diversity, whereas teaching experience does not. These results suggest the importance of training in attention to diversity to cover pupils' needs in class.

Keyword: Inclusive education

\section{Introducción}

En 1975, la Convención sobre los derechos de las personas con discapacidad de Naciones Unidas declaró que se debe "promover, proteger y asegurar el goce pleno y en condiciones de igualdad de todos los derechos humanos y libertades fundamentales de todas las personas con discapacidad, y promover el respeto de su dignidad inherente" (Naciones Unidas, 2006, art. 1). No obstante, no fue hasta 1994 cuando la Declaración de Salamanca y Marco de Acción para las necesidades educativas especiales adoptó el concepto de "educación inclusiva", que promovía oportunidades de aprendizaje personalizadas basadas en la equidad, en lugar de en la igualdad (Smyth, Shevlin, Buchner, Biewer, Flynn, Latimier, Šiška, Toboso-Martín, Rodríguez Díaz. y Ferreira, 2014; UNESCO, 2005). En 2006, la Convención sobre los derechos de las personas con discapacidad estableció en su artículo 24 "el derecho de las personas con discapacidad a la educación" (Naciones Unidas, 2006, art. 24); consecuentemente, la 
escuela debe garantizar la educación general teniendo en cuenta la diversidad de su alumnado con la finalidad de desarrollar sus habilidades y su dignidad en todos los aspectos de su vida.

La educación inclusiva proporciona un acceso útil y significativo al currículo general a estudiantes que necesitan apoyo especial o atención individualizada (Hagiwara et al., 2019). De esta forma, la educación inclusiva se manifiesta en la escuela cuando los estudiantes con discapacidad aprenden y participan en el contexto general de educación junto con sus compañeros de la misma edad cronológica y al mismo tiempo que estos (Amor et al., 2018, citado en Hagiwara, Shogren, Thompson, Burke, Uyanik, Amor, Vergudo y Aguayo, 2019). Este proceso de enseñanza individualizada está estrechamente relacionado con el concepto de "atención a la diversidad" y su finalidad de enseñar bajo el principio de equidad. Asimismo, tiene como objetivo comprender las características de los estudiantes para ajustar las prácticas docentes a sus necesidades. Este concepto se ha asociado a la comparación entre estudiantes con discapacidad y sin ella, proporcionando a los primeros más recursos que eviten el fracaso escolar. Sin embargo, la atención a la diversidad persigue eliminar las posibles barreras que imposibilitan que todo el alumnado alcance los objetivos educativos y su derecho a una educación de calidad (Echeíta, 2005). Por lo tanto, la atención a la diversidad involucra a estudiantes con bajos niveles de motivación, sin habilidades sociales, con cualquier tipo de discapacidad o trastorno, niños migrantes cuya lengua nativa difiere de la lengua hablada en el país de destino, etc. (Gentile, 2002, citado en Echeíta, 2005), con la finalidad de enriquecer el acceso al currículo general considerando todos los contextos posibles y diversos y actuando de acuerdo con ellos (García Rubio, 2017).

El principal objetivo de la presente investigación es indagar sobre la concepción de los maestros y maestras de Educación Infantil y Primaria de las aulas madrileñas sobre la atención a la diversidad y sobre su capacidad para aplicarla en el contexto real. Los resultados se extraerán del análisis de cuestionarios dirigidos a conocer la formación de los docentes en atención a la diversidad, la manera en que organizan su docencia, a qué tipo de metodologías recurren para cubrir las necesidades de su alumnado y sus opiniones sobre este tema en diferentes contextos educativos.

\section{Atención a la diversidad: contextualización}

El concepto de atención a la diversidad se basa en la responsabilidad de todo sistema educativo de asegurar el derecho fundamental a la educación de cada niño. Debe reconocer las necesidades de los estudiantes, evitando la inequidad y garantizando un proceso de aprendizaje abierto y flexible y una educación de calidad para todos, según Arenque y Barrio de la Puente (2010). Estos autores establecen que un proceso de aprendizaje exitoso es posible para todos los niños si se siguen dichas directrices. Por lo tanto, Arenque y Barrio de la Puente (2010) asocian la atención a la diversidad con la aplicación de un modelo educativo que se adapte al contexto de los estudiantes con la finalidad de proporcionar ayuda pedagógica personalizada y adaptaciones de las prácticas docentes a sus capacidades, motivaciones e intereses.

Además, Echeíta (2005) propone una definición de atención a la diversidad conectada a la ecología de la educación, describiéndola como la comprensión y la actitud respetuosa hacia la pluralidad de cada estudiante. Esta es la única manera de satisfacer las necesidades de todos y de lograr los objetivos propuestos para ellos. Por tanto, el principal propósito de la atención a la diversidad es individualizar el proceso de aprendizaje en la escuela. Si los docentes adaptan su enseñanza, modificando objetivos, contenidos, criterios de evaluación, etc., ningún estudiante perdería la oportunidad de aprender. Por consiguiente, la atención a la diversidad podría 
considerarse como la forma de trabajar con equidad y de compensar inequidades y desventajas entre estudiantes.

Achinstein y Athanases (2005) enfatizan la importancia de comprender el concepto de "equidad" y su relación con la atención a la diversidad. Enseñar bajo el paraguas de la equidad asegura que todo el alumnado sea provisto de recursos y atención personalizados para apoyar su proceso de aprendizaje. Por consiguiente, trabajar para la equidad no solo implica el desarrollo de competencias académicas, sino también el desarrollo de la competencia cultural. Por tanto, los maestros y maestras podrían conocer mejor el contexto de sus estudiantes si incluyesen prácticas docentes basadas en la equidad, no solo en relación con su situación en casa o sus capacidades y limitaciones, sino también con su lugar de nacimiento. Esta es la forma adecuada de trabajar la diversidad en el aula y sacar provecho de ella (Ladson-Billings, 2001, citado en Achinstein \& Athanases, 2005).

Por otro lado, un sistema educativo basado en la reducción de la exclusión social debida a desventajas socioculturales, o a cualquier tipo de discapacidad o trastorno, puede ser considerado como un sistema que trabaja para asegurar una educación inclusiva (Arenque y Barrio de la Puente, 2010). Este modelo educativo se basa en el mismo principio de la educación regular o estándar: todos los niños deben aprender juntos aunque hayan nacido en diferentes países o presenten capacidades distintas (Booth, 2000, citado en Molina, 2015). Sin embargo, algunos investigadores han llegado a la conclusión de que ciertas prácticas docentes basadas en el modelo de educación estándar pueden promover actitudes de discriminación y de exclusión en el aula. Por ello, los docentes deben ser conscientes de los contextos y las características personales de su alumnado y actuar de acuerdo con sus necesidades para proporcionar una educación apropiada que reduzca la inequidad y fomente oportunidades de aprendizaje y procesos de evaluación adecuados (Foutoul y Fierro, 2011, citado en Molina, 2015).

Por otra parte, los docentes deberían reconocer la importancia de planificar su docencia para satisfacer las necesidades del alumnado y alcanzar los objetivos de la educación inclusiva. No obstante, cuando programan su actividad docente, crean una sección específica y diferenciada para alumnos con necesidades educativas específicas (NEE) llamada "Atención a la diversidad", destinada a dar respuesta a las dificultades de aprendizaje que presentan estudiantes con discapacidad y trastornos, con altas capacidades o migrantes cuya lengua nativa difiere del español (Arjona Fernández, 2010). Esta concepción no representa el verdadero objetivo de la atención a la diversidad.

Según Roselló Ramón (2010), un plan docente debería considerarse como un proceso de reflexión para comprender el contexto del aula, porque cuanto más sepamos sobre nuestros alumnos y alumnas, mejor serán las estrategias educativas implementadas para crear oportunidades de aprendizaje para todos. Como resultado, la planificación de sesiones con diferentes niveles deberá desaparecer. Así pues, una programación didáctica se definiría como el proceso de desarrollar unidades didácticas durante un periodo de tiempo específico y para un grupo en concreto (García Vidal, González Manjón y Herrera Lara, 2005). Esta debería incorporar varias secciones que aseguren que los estudiantes alcanzan los objetivos propuestos. Siguiendo una perspectiva inclusiva, la educación especial debería incluirse en la educación general, desarrollando un plan de intervención guiado por especialistas del campo de la Pedagogía Terapéutica (PT) y de Audición y Lenguaje (AL), para dar respuesta a las dificultades del alumnado (Álvarez Duran, 2009). 
Por consiguiente, la programación y la metodología del docente deberían modificarse para adaptarse a los fines de la educación inclusiva, siguiendo las directrices del Diseño Universal para el Aprendizaje (DUA) (Roselló Ramón, 2010) o la co-docencia ${ }^{1}$ entre otros.

\section{Investigaciones previas}

La evaluación de las actitudes del profesorado hacia la atención a la diversidad en la escuela es, desde hace un tiempo, un campo de interés en la investigación educativa. Como ejemplo, Sales, Moliner y Sanchiz (2001) realizaron un estudio sobre las actitudes de los docentes hacia la atención a la diversidad para el que diseñaron un cuestionario con dimensiones como "actitudes y dinámica de aula", "estrategias para la atención a la diversidad" o "necesidades formativas para atender a la diversidad".

En la misma línea, Bravo (2013) desarrolló una escala para medir las opiniones de los docentes sobre la educación inclusiva en Cartago, Costa Rica. Este instrumento se dividía en tres sub-escalas: "Fundamentos, Condiciones y Actuaciones". La muestra estaba compuesta por 614 maestros y maestras de Educación Primaria cuya formación académica se limitaba mayoritariamente a su grado universitario $(61 \%)$ y su experiencia laboral era de entre 9 y 30 años. Los participantes presentaban opiniones favorables respecto de la educación inclusiva. Sin embargo, estaban parcialmente en desacuerdo cuando se les preguntó por la inclusión de estudiantes con NEE en el aula ordinaria. Por el contrario, mostraban acuerdo en el hecho de que la educación inclusiva ayuda a crear una sociedad inclusiva. Asimismo, el 56\% de la muestra pensaba que la educación inclusiva presenta más ventajas que desventajas. Por otra parte, los docentes señalaron que no contaban con los recursos, materiales y formación suficientes para garantizar una educación de calidad a alumnado con NEE. Finalmente, los participantes eran conscientes de la necesidad de prácticas inclusivas para garantizar la atención a la diversidad, de la reducción del número de alumnos por aula y de la colaboración entre docentes y especialistas en el área para cubrir las necesidades del alumnado.

Otro ejemplo de investigación es el de Nogales (2012), que llevó a cabo un estudio que buscaba demostrar si el conocimiento por parte de los docentes sobre la atención a la diversidad tenía relación con sus actitudes y prácticas en educación inclusiva. Para ello, se empleó un cuestionario que completaron 61 maestros y maestras de Educación Primaria con edades comprendidas entre los 31 y los 60 años. La experiencia laboral de la muestra oscilaba entre menos de 10 años y más de 25 . El $21.3 \%$ de los participantes reconocía no haber recibido ningún tipo de formación en atención a la diversidad, aunque admitía su importancia para el desarrollo de prácticas inclusivas y para cubrir las necesidades del alumnado (80.33\%). Igualmente, se mostraba acuerdo en la falta de formación en atención a la diversidad durante sus estudios superiores. A pesar de conocer sus limitaciones en esta área de conocimiento, el $91.8 \%$ de los docentes afirmaban crear material personalizado para alumnos con NEE, y el $50.82 \%$ manifestaban trabajar de manera coordinada con docentes especializados en NEE. Por otra parte, un $65.57 \%$ de la muestra creía que el equipo directivo debería involucrarse más en el desarrollo de actitudes y prácticas inclusivas en la escuela.

Sales et al., (2001), Nogales (2012) y Bravo (2013) descubrieron que los docentes reconocían sus limitaciones en formación sobre atención a la diversidad. Por esta razón, Cejudo, Díaz, Losada y Pérez-González (2016) analizaron la importancia de los aspectos que definen esta dimensión y por qué los docentes necesitan dominar este campo. Su muestra de estudio estaba compuesta por 181 docentes de edades comprendidas entre los 24 y los 65 años, de los cuales 52 eran maestros de Educación Infantil y 129 de Educación Primaria. Los datos se recogieron mediante la "Escala de 
Necesidades Formativas sobre Atención a la Diversidad" (ENFAD), compuesta por dos subescalas relacionadas con la importancia y la necesidad de este tipo de formación. Al igual que ocurrió con anteriores estudios, Cejudo et al. (2016) confirmaron que los docentes mostraban preocupación por su formación académica en atención a la diversidad, haciendo hincapié en casos de estudiantes con problemas de comportamiento. Finalmente, los maestros de Educación Primaria admitían necesitar más formación en atención a la diversidad que los de Educación Infantil.

Algunos años después, Azorín (2017) llevó a cabo una revisión bibliográfica en la que evaluó 24 cuestionarios realizados entre 2002 y 2014 y su fiabilidad para medir este asunto. Azorín (2017) detectó cinco categorías presentes en todos los instrumentos de análisis (aparte de las variables sociodemográficas para describir la muestra):

- medidas de atención a la diversidad,

- actitudes hacia la atención a la diversidad,

- opiniones sobre la educación inclusiva,

- formación en materia de atención a la diversidad,

- $\quad$ prácticas educativas y sociales, y

- $\quad$ voces de las familias. (Azorín, 2017, p.1047).

Estas variables son útiles para analizar las actitudes de los docentes hacia la atención a la diversidad de manera profunda y para clasificar las respuestas con el fin de obtener conclusiones fiables.

En suma, la atención a la diversidad está relacionada con proporcionar a cada estudiante, con discapacidad o sin ella, el apoyo necesario. Es un proceso que debería entenderse como la adaptación de las escuelas a las necesidades de los estudiantes con la finalidad de garantizar una formación personalizada para desarrollar las capacidades del niño al máximo. Sin embargo, si la comunidad educativa aun confunde inclusión con integración, la atención a la diversidad no puede garantizarse. De hecho, sería entendida como una forma de clasificación de los estudiantes dependiente de sus capacidades, algo que no es el objetivo de la atención a la diversidad. Esta última idea es el motivo del presente estudio: comprender las respuestas de los docentes en relación con la atención a la diversidad y la educación inclusiva desde perspectivas educativas diferentes, como su formación o sus estrategias didácticas en el aula.

\section{Preguntas de investigación}

Este estudio pretende indagar en las opiniones y creencias sobre la atención a la diversidad de maestros y maestras de Educación Infantil y Primaria. Para ello, partimos de las siguientes preguntas de investigación:

- $\quad$ ¿Existe una diferencia significativa en las concepciones de los docentes sobre la atención a la diversidad y la educación inclusiva dependiendo de sus características sociodemográficas (sexo, edad, experiencia laboral, etc.) y de su formación académica?

- ¿QQué tipo de opiniones sobre la atención a la diversidad encontramos entre los maestros y maestras de Educación Infantil y Primaria?

- ¿ ¿Puede que los docentes de Educación Infantil sean más conscientes de la importancia de la educación inclusiva que los de Primaria o viceversa? 


\section{Metodología}

El presente estudio ha seguido una metodología cuantitativa basada en un "descriptive study of populations through survey research with probability sampling" (Montero y León, 2007 , p. 850). Para ello, se utilizará un cuestionario y un enfoque ex post facto, ya que la finalidad es describir una población particular de docentes sin una hipótesis clara previa (Montero y Leon, 2007). El diseño del cuestionario se basa, por una parte, en diferentes variables sociodemográficas para describir la muestra y, por otra, en cuatro dimensiones educativas relacionadas con la atención a la diversidad: formación recibida, forma de programar, metodologías y opiniones sobre la atención a la diversidad (Anexo I), siguiendo estudios previos (Azorín, 2017; Sales et al., 2001).

El cuestionario está formado por siete variables sociodemográficas y 21 preguntas repartidas entre las cuatro dimensiones mencionadas. Las respuestas se presentaron utilizando una escala Likert de 6 puntos. Para conseguir el objetivo de la investigación, los participantes deben posicionarse ideológicamente de manera clara. Por ello, un número par de posibles respuestas es el adecuado, evitando posiciones intermedias de una escala Likert impar. Las respuestas oscilan entre "Totalmente de acuerdo/Totalmente en desacuerdo", "Nunca/Siempre" y "Sí/No".

Una vez diseñado el cuestionario online, este fue enviado por correo electrónico, junto con una carta de presentación, al equipo directivo de 120 colegios de la Comunidad Autónoma de Madrid (CAM), explicando la finalidad de la investigación y solicitando su participación en el estudio. Los centros fueron seleccionados de manera aleatoria, manteniendo un equilibrio entre centros públicos, concertados y privados.

Las respuestas de los docentes fueron recopiladas en una hoja de cálculo, cuyos datos se volcaron posteriormente en una base de datos diseñada con el programa de análisis cuantitativo SPSS statistics software para el análisis de estos.

\section{Resultados y discusión}

Este apartado se divide en dos secciones: por una parte, la descripción de la muestra mediante estadística descriptiva; por otra, la respuesta a las preguntas de investigación recurriendo a la estadística inferencial. Dichos resultados se compararán con estudios previos.

\section{Descripción de la muestra}

Esta investigación tiene como finalidad analizar la visión de los maestros y maestras sobre la atención a la diversidad y cómo estos organizan su práctica docente para cubrir las necesidades del alumnado en la escuela. Para ello, 103 docentes de la CAM respondieron a un cuestionario que indagaba sobre dichos asuntos. La muestra se compone de 91 mujeres (88.3\%) y 12 hombres (11.7\%). Este desequilibrio entre sexos se explica observando los datos de la comunidad: durante el curso académico $2017 / 2018$, de un total de 51.712 docentes, 44.022 eran maestras $(85.1 \%)$ y 7.690 eran maestros (14.9\%) (Ministerio de Educación y Formación Profesional, 2019). Por consiguiente, la muestra representa a la población docente en relación con su distribución por sexos en la CAM.

La mayoría de los participantes tenían entre 31 y 40 años $(n=31 ; 30.1 \%)$, seguidos por el grupo de edad de 41 a 50 años ( $n=28 ; 27.2 \%$ ). De los restantes, 22 participantes eran menores de 31 y otros 22 docentes eran mayores de 51 (Figura 1). 


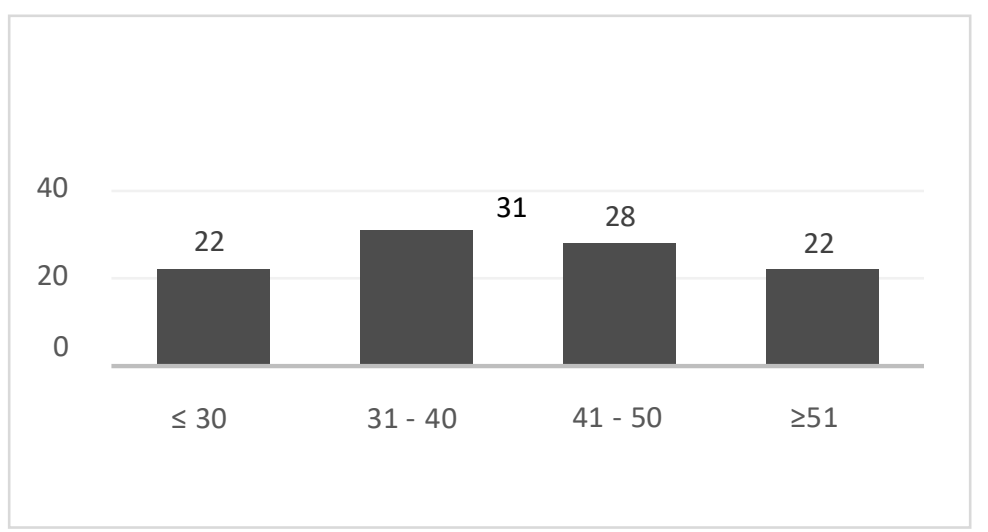

Figura 1. Grupos de edad en la muestra

Los participantes se dividieron en dos grupos según su grado universitario: Educción Infantil y Educación Primaria. Sin embargo, la formación académica de algunos de los participantes era más amplia. Para analizar su formación académica completa, respondieron a preguntas relacionadas con su especialización y sobre si poseían otros estudios universitarios además del grado. Los maestros de Educación Infantil suponen un total del $32 \%$ de la muestra $(n=33)$ y los 70 restantes trabajan en Educación Primaria (68\%). Entre ellos, 19 docentes son especialistas en ambas etapas educativas, ya que poseen los dos grados universitarios.

Adicionalmente, la muestra fue analizada cruzando los datos sobre los grupos de edad y su formación académica. Según este criterio, el $64.1 \%$ de los docentes no tiene más formación aparte de su grado universitario; por el contrario, el grupo de edad más joven $(n=22)$ es el que más estudios ha realizado (14 de $22 ; 63.6 \%)$. Más de la mitad de la muestra, que va de los 31 a los 40 y de los 41 a los 50 años, no tiene formación más allá de su grado universitario (Figura 2).

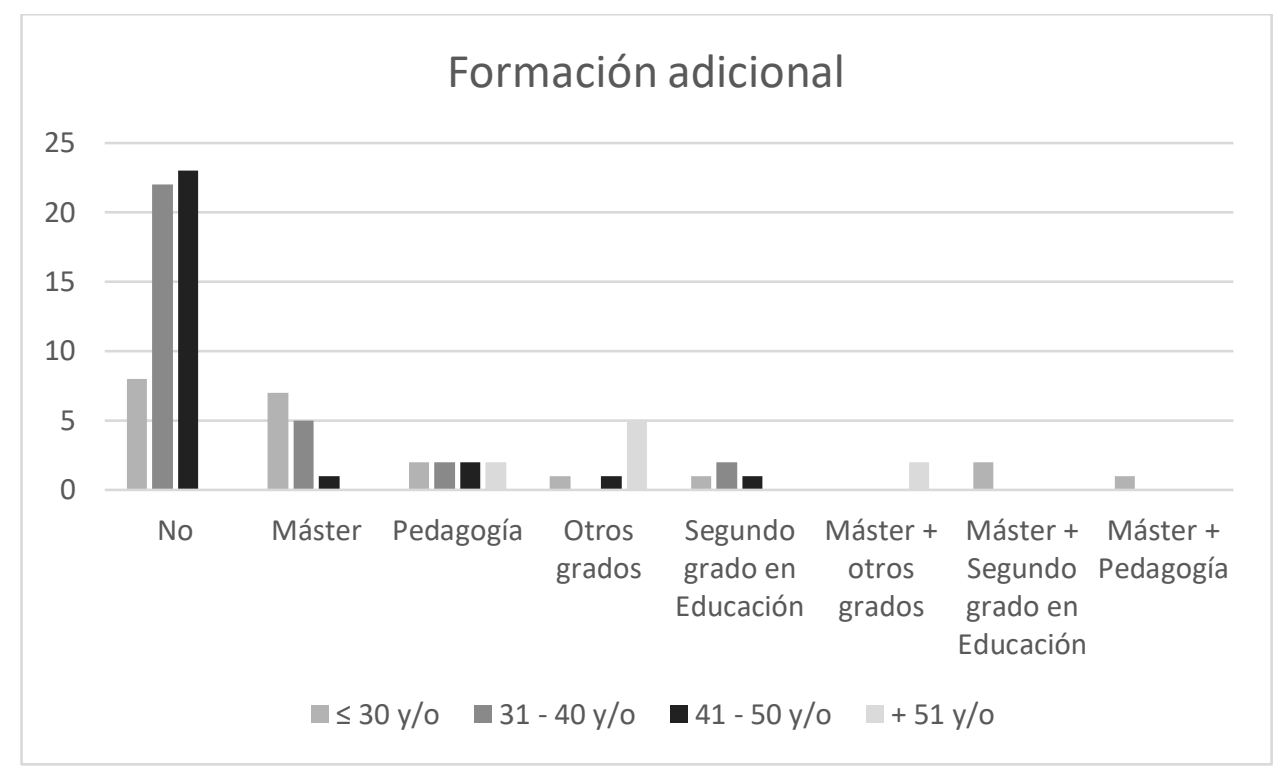

Figura 2. Formación adicional de los docentes de la muestra

Si consideramos la especialización como criterio para describir a los participantes del presente estudio (Figura 3), se pueden observar resultados similares, ya que el $42.7 \%$ no tiene ninguna especialización. Además, en términos generales, los docentes más 
experimentados son los que menos estudios adicionales han realizado tras finalizar el grado universitario. La especialización en Inglés es la más común (19.4\%), cifra que contrasta con las relativas a las áreas de educación especial (PT y AL), que muestran menos impacto en la muestra $(n=12)$.

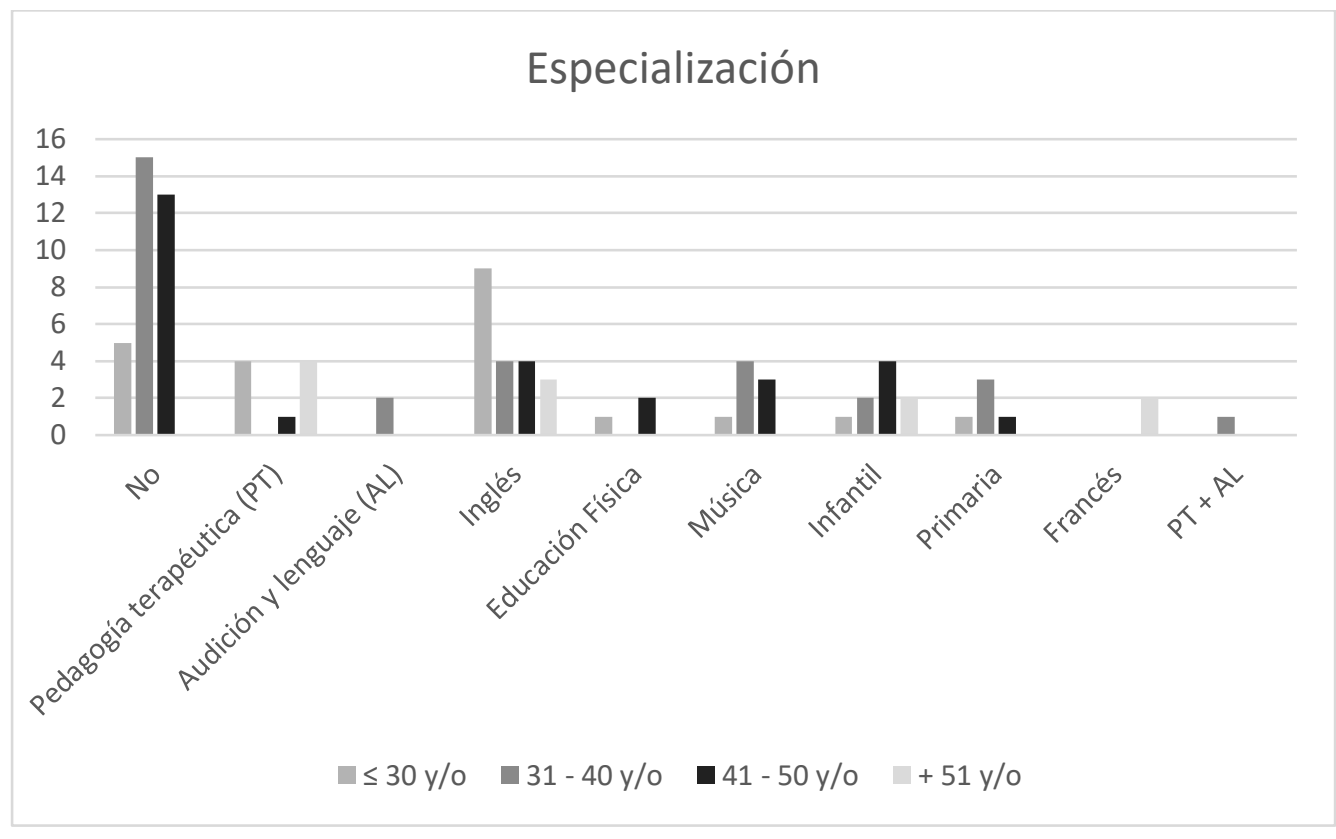

Figura 3. Especialización de los decentes de la muestra

Por otra parte, se consultó a los participantes sobre sus años de experiencia laboral. Estos podían elegir entre ocho intervalos de cinco en cinco años. La mayoría de los docentes muestran una experiencia laboral de entre 1 y 5 años $(n=25)$, a pesar de que la mayoría se sitúa en el rango de edad de entre 31 y 40 años (Figura 4).

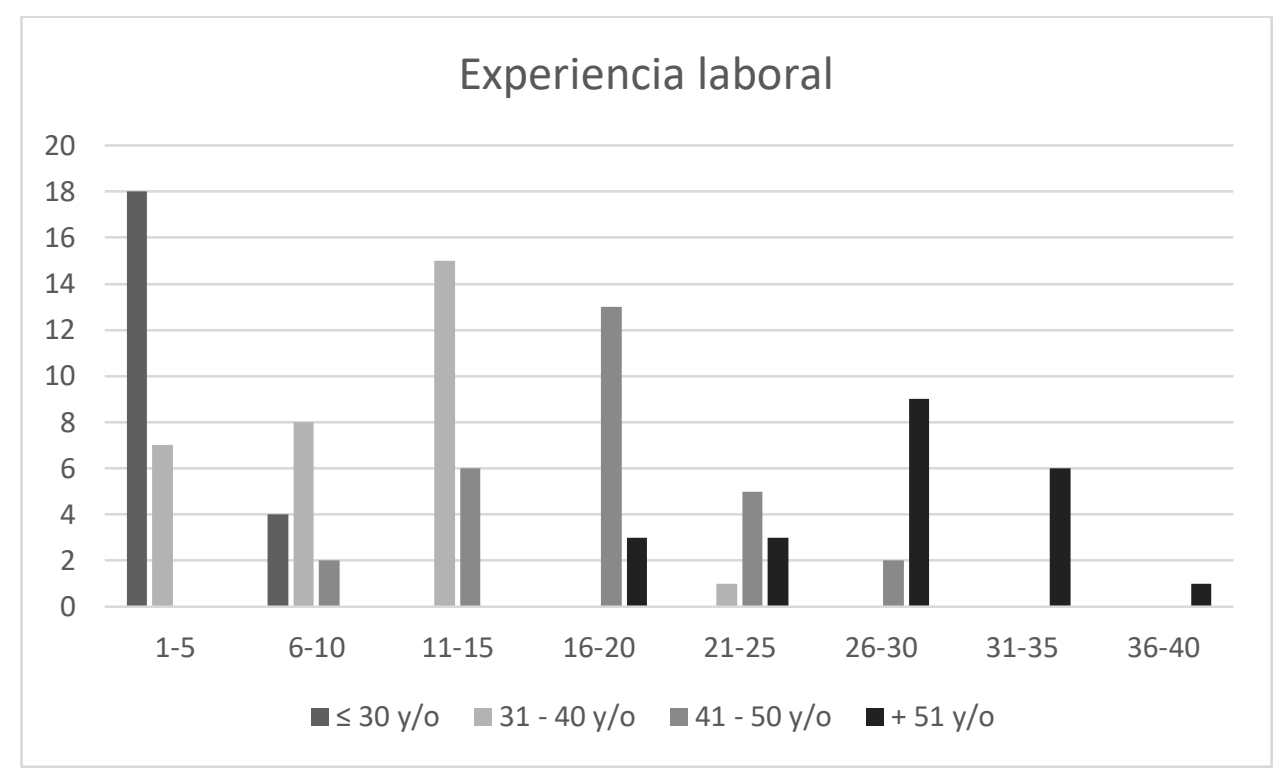

Figura 4. Experiencia laboral en años de la muestra

Se habría esperado que la experiencia laboral de la muestra fuera mayor de cinco años, pero siete docentes de 31 (22.5\%), con edades comprendidas entre los 31 y los 40 años, han estado trabajando como maestros y maestras durante un periodo de tiempo 
relativamente breve. De cualquier manera, la distribución de las cifras es coherente en términos generales, puesto que estas incrementan con la edad de los participantes.

Finalmente, aunque el cuestionario fue enviado a centros públicos, concertados y privados en la misma proporción, la respuesta de los dos últimos tipos de centros fue muy inferior, por lo que la variable "tipo de centro" se descartó para el análisis por falta de representatividad.

\section{Respuesta a las preguntas de investigación}

La atención a la diversidad debe considerarse la base para asegurar procesos de aprendizaje de calidad en toda etapa educativa. Las administraciones, junto con los proyectos de los centros, los equipos directivos y los docentes, son responsables de alcanzar este objetivo. Por esta razón, la perspectiva de los maestros y maestras de Educación Infantil y Primaria sobre este asunto tiene una relevancia esencial si queremos saber qué ocurre en las aulas en relación con la educación inclusiva. Para responder a las preguntas de investigación, se aplicarán pruebas de estadística inferencial a los datos obtenidos de las respuestas al cuestionario.

Aunque la muestra supera los 30 participantes, la prueba de Chi-cuadrado daba como resultado valores menores que 5 para cada variable. Por lo tanto, se aplicó la prueba de bondad de ajuste Kolmogorov-Smirnov para determinar si la muestra presentaba una distribución normal en relación con las dimensiones del cuestionario. En este caso, las cuatro dimensiones dieron un resultado de $p<.05$. Consecuentemente, la muestra no presenta una distribución normal, por lo que se usaron pruebas no paramétricas. El análisis se llevó, pues, a cabo mediante la prueba de Kruskal-Wallis para comparar más de dos grupos o submuestras y mediante la prueba $U$ de Man-Whitney para comparar dos únicos grupos o muestras independientes.

\section{Concepción de la atención a la diversidad entre mujeres y hombres}

La primera pregunta que busca responder este estudio es si existen diferencias de concepción sobre la atención a la diversidad entre hombres y mujeres. Como se ha mencionado, la muestra está compuesta por 91 mujeres y 12 hombres, siendo este desequilibrio representativo de la población total de maestros y maestras en la CAM. La prueba de Kruskal-Wallis no muestra resultados significativos en tres de las cuatro dimensiones. No obstante, para la dimensión "Opiniones sobre la atención a la diversidad" se obtuvo un resultado de $p<.01$ (con un valor de distribución asintótica de .083).

Tras este resultado, la variable "sexo" se cruzó con esta dimensión usando la prueba U de Man-Whitney, lo que confirmó una diferencia de rangos en las preguntas $17(p=.013)$, $19(p=.004)$ y $20(p=.049)$. La pregunta 17 plantea si deben ser los docentes especializados en educación especial y los equipos directivos quienes deben encargarse de la inclusión del alumnado con NEE en el aula. Los maestros mostraron opiniones desiguales dada la dispersión de los datos en el rango promedio ${ }^{2}(\mathrm{RP})$ de esta variable ( $\mathrm{RP}=71.54)$, mientras que el $\mathrm{RP}$ en las respuestas de las maestras es de $\mathrm{RP}=49.42$. Es más, la pregunta 19 plantea que los alumnos con NEE deberían ser atendidos por docentes especializados en NEE, y los resultados fueron similares. Los maestros mostraron un rango promedio de $\mathrm{RP}=74.75$, mientras que las maestras mostraron un mayor acuerdo en sus respuestas $(\mathrm{RP}=49.00)$. Por último, la pregunta número 20 especifica que las familias deberían trabajar junto con la escuela para facilitar la inclusión del alumnado con NEE. En este caso, los maestros muestran un rango promedio más bajo. Bravo (2013) presentó resultados similares, ya que su muestra 
indicaba que los docentes estaban parcialmente de acuerdo cuando se les preguntó sobre la inclusión de alumnado con NEE en el aula ordinaria. Por consiguiente, se concluye que en la dimensión "Opinión sobre la atención a la diversidad" existen diferencias entre hombre y mujeres en su concepción de la gestión de la atención a la diversidad, especialmente para las variables relacionadas con el tratamiento del alumnado con NEE en la escuela.

\section{La edad de los docentes influye en su comprensión de la atención a la diversidad}

La prueba de Kruskal-Wallis se utilizó para verificar si la edad es un factor determinante en la comprensión de la atención a la diversidad en las cuatro dimensiones. Sin embargo, la prueba no produjo resultados significativos entre los grupos de edad, excepto para la dimensión "Forma de programar" $(p=.059)$. El grupo menor de 30 años mostraba diferencias significativas en el rango promedio $(\mathrm{RP}=44.99)$ cuando los resultados eran comparados con los de los otros grupos (valores entre 51 y 55). Además, la prueba de Kruskal-Wallis se usó para especificar diferencias significativas entre los grupos de edad y las preguntas de dicha dimensión. Estas diferencias se encontraron en las preguntas $6(p=.001)$ y $7(p=.042)$. La primera de ellas buscaba saber si los docentes organizan su enseñanza al principio del curso académico; la segunda estaba relacionada con la frecuencia con la que estos programan cada unidad didáctica. Las respuestas a ambas preguntas confirman que los docentes más jóvenes muestran una menor diferencia en el rango promedio, por lo que programan su docencia más habitualmente que el resto de grupos de edad.

Arjona Fernández (2010) señala que la planificación docente es esencial para cubrir las necesidades del alumnado. Asimismo, Roselló Ramón (2010) establece que esta práctica debe considerarse como un proceso reflexivo para comprender el contexto del aula con el fin de implementar estrategias de enseñanza personalizadas. Es posible que los docentes más jóvenes sean más conscientes de esto debido a que su formación está más actualizada. También se puede tener en cuenta que, como se mostró en el Grafico II, este grupo de edad tiene un grado de formación superior. Es posible que esta formación adicional influya en la manera en la que organizan su docencia y sean más conscientes de la importancia de programar su práctica en el aula. Consecuentemente, podemos admitir una diferencia significativa entre grupos de edad en lo relativo a la dimensión "Forma de programar" para atender la diversidad.

La experiencia laboral influye en la concepción sobre la atención a la diversidad

Se podría dar por hecho que cuanto más experimentado es el docente, mejor y antes serán detectadas y cubiertas las necesidades del alumnado. Por ello, se esperaba que los docentes con mayor experiencia laboral mostraran resultados diferentes en las cuatro dimensiones que aquellos con menos experiencia. Sin embargo, los ocho grupos creados para clasificar la experiencia laboral obtuvieron valores similares en su rango promedio. Asimismo, la prueba de Kruskal-Wallis produjo un valor $\mathrm{p}>.05$ para distribuciones asintóticas en las cuatro dimensiones. Por lo tanto, en este caso se asume que, según nuestra muestra, la experiencia laboral no es un factor que afecte a la concepción sobre la atención a la diversidad.

Cuanto mayor sea el nivel educativo del docente, mejor será su concepto sobre la atención a la diversidad

Varios autores, como Azorín (2017), Bravo (2013) o Sales et al. (2001), muestran su acuerdo sobre la importancia de la formación en atención a la diversidad para atender las necesidades del alumnado. La prueba de Kruskal-Wallis verificó que existen 
diferencias significativas entre la variable sociodemográfica "formación académica" y las dimensiones "Formación recibida", con un valor de $p<.05$, y "Opinión sobre la atención a la diversidad", con un valor de $p<.01$.

La prueba de Kruskal Wallis se utilizó de nuevo para especificar qué preguntas de ambas dimensiones proporcionaban los valores significativos. La primera dimensión daba resultados significativos en todas las peguntas (Tabla 1). La pregunta 4 basada en la auto-evaluación del profesorado sobre su actuación con alumnado con NEE dio un resultado de $\mathrm{p}=.038$ y el grupo de docentes con una mayor diferencia en su rango promedio se corresponde con aquellos que estudiaron un grado pedagógico. Esto se puede explicar entendiendo que están mejor preparados en este campo y proporcionan una mayor variedad de opiniones en esta dimensión. La pregunta 5 se centra en la autoevaluación en relación con la formación en atención a la diversidad $(p=.032)$. Los docentes con grados pedagógicos obtuvieron la mayor diferencia en el rango promedio de nuevo ( $\mathrm{RP}=68.13)$, hecho que se puede explicar utilizando los mismos argumentos que en el caso anterior.

Tabla 1. Prueba de Kruskal-Wallis

\begin{tabular}{|c|c|c|c|c|c|}
\hline \multicolumn{6}{|c|}{ Variable "Formación académica" y dimensión "Formación recibida" } \\
\hline & Pregunta 1 & Pregunta 2 & Pregunta 3 & Pregunta 4 & Pregunta 5 \\
\hline Chi-cuadrado & 13.839 & 15.208 & 15.732 & 14.841 & 15.356 \\
\hline Gl & 7 & 7 & 7 & 7 & 7 \\
\hline $\begin{array}{l}\text { Distribución } \\
\text { asintótica }\end{array}$ & .054 & .033 & .028 & .038 & .032 \\
\hline
\end{tabular}

En contraste, la dimensión "Opinión sobre la atención a la diversidad" solo arrojó resultados significativos en la pregunta $16(p=.055)$, que especifica que la educación inclusiva fomenta la integración social para alumnado con NEE.

Finalmente, se concluye que los docentes que no tienen formación académica más allá de su grado universitario (Educación Infantil o Primaria, sin otros títulos adicionales) presentan una diferencia en el rango promedio mayor que aquellos docentes con una formación más amplia. Además, los docentes con grados pedagógicos son los que sobresalen positivamente en esta dimensión.

La especialización influye en la forma en que los docentes entienden la atención a la diversidad

Siguiendo una propuesta similar a la de la hipótesis previa, cuanto más conocimiento posean los docentes sobre la atención a la diversidad, más personalizada será su docencia. Se supone que los docentes especializados en PT o AL presentarán resultados diferentes que aquellos especializados en Música o en Inglés. En primer lugar, la prueba de Kruskal-Wallis confirmó que la variable "especialización docente" correlaciona significativamente con la dimensión "Formación recibida" $(p=.015)$. Aunque las otras tres dimensiones no correlacionan, ofrecen resultados notables que destacaremos a continuación.

Tras aplicar la prueba de Kruskal-Wallis a la dimensión "Formación recibida" cruzada con la variable "especialización docente", las preguntas 1 y 3 produjeron diferencias significativas $(p<.01)$. Los especialistas en PT mostraron una menor diferencia en el 
rango promedio $(\mathrm{RP}=16.39)$ cuando se les preguntó sobre la formación que habían recibido en atención a la diversidad. Este hecho tiene sentido, ya que son responsables de la enseñanza a alumnado con NEE en la escuela. Por otra parte, los maestros y maestras de Música coincidían en la necesidad de incrementar su formación en este campo $(R P=38.88)$, seguidos por los especialistas en Francés $(R P=34.75)$ y en Inglés $(\mathrm{RP}=29.85)$.

Aunque la dimensión "Opinión sobre la atención a la diversidad" no presenta diferencias significativas, hay algunas declaraciones que merecen atención. Por ejemplo, los docentes de Educación Física son lo que muestran una visión más amplia de la atención a la diversidad. Esto se puede explicar debido a que esta área educativa ha sido tradicionalmente más consciente de las discapacidades físicas, y en su formación como maestros y maestras se ha dedicado más tiempo a la atención a las necesidades específicas del alumnado. Por otra parte, los especialistas en lenguas extranjeras han presentado la mayor dispersión en el rango promedio en todas las dimensiones. De ello se extrae que los docentes han recibido formación relativa a su especialidad, descuidando así las estrategias de inclusión en algunos currículos académicos. En el caso de los docentes de lenguas extranjeras, la atención a la diversidad supone un doble esfuerzo: no solo deben saber cómo enseñar de una manera inclusiva, sino que además deben hacerlo usando como medio de comunicación una lengua diferente a la lengua nativa del alumnado. Esto supone no solo solventar problemas de comprensión del contenido, sino también dificultades de comunicación.

Tras la explicación de los resultados, podemos asumir que la especialización influye en la atención a la diversidad, ya que los docentes de PT son más conscientes de la importancia de esta que otros especialistas.

¿Son los docentes de Educación Infantil más conscientes de la importancia de la educación inclusiva que los de Primaria o viceversa?

Finalmente, la etapa educativa (Infantil o Primaria) puede influir en la percepción de los docentes sobre la atención a la diversidad. Para averiguarlo se aplicó la prueba de Kruskal-Wallis, que produjo resultados significativos en la dimensión "Opinión sobre la atención a la diversidad" $(p=0.37)$. Así, se aplicó la prueba $U$ de Man-Whitney para identificar el motivo de la diferencia entre grupos. La pregunta 18 arrojó una $p=.058$ y los maestros y maestras de Educación Infantil consideraban, en mayor medida que los de Primaria, que las familias son clave para trabajar la inclusión.

Por otra parte, Cejudo et al. (2016) señalaron que los docentes de Educación Primaria reconocían la necesidad de más formación en atención a la diversidad que los de Educación Infantil. Aunque la dimensión "Formación recibida" no dio valores $p<.05$, la prueba U de Man-Whitney se usó para verificar las conclusiones de Cejudo et al. (2016). Sin embargo, en la presente muestra ambos grupos de docentes mostraron percepciones similares sobre su formación en esta área, coincidiendo en su insuficiencia a lo largo de su etapa universitaria. Por ello, concluimos que no existen diferencias entre los docentes de ambas etapas educativas, ya que ambos grupos mostraron resultados similares ante las preguntas sobre su percepción de la atención a la diversidad.

\section{Conclusiones}

Este estudio examina las respuestas sobre atención a la diversidad de docentes de Educación Infantil y Primaria de distintos contextos educativos mediante un cuestionario diseñado para este propósito con la finalidad de analizar la situación actual en las escuelas de la CAM. 
Tras analizar los resultados y compararlos con estudios previos similares, nuestros hallazgos sugieren que la formación en atención a la diversidad durante y con posterioridad a la finalización de los estudios universitarios es un factor clave para fomentar una educación inclusiva en el aula de manera eficaz. Sin embargo, la mayoría de los docentes de nuestra muestra reconocen sus limitaciones y su falta de formación en atención a la diversidad a lo largo de su periodo universitario. Por otra parte, los docentes más jóvenes son los que más grados o cualificación extra poseen para complementar su grado universitario. Estos parecen ser más conscientes de las tendencias en atención a la diversidad, especialmente aquellos que han cursado grados pedagógicos.

Es más, cada especialización educativa implica una concepción diferente de la atención a la diversidad. Aunque la mayoría de los participantes no cuentan con una especialización, y la mayor parte de los especialistas pertenecen a la rama de inglés como lengua extranjera, los especialistas en PT mostraron los resultados más positivos, hecho que es coherente con sus funciones diarias en la escuela. En contraste, los especialistas en lengua extranjera son los que presentaron peores resultados en las cuatro dimensiones del cuestionario. Este hecho se puede explicar debido a que su formación académica se concentra en la didáctica de la lengua extranjera y a que deben realizar un doble esfuerzo en el aula al tener que comunicarse en una lengua distinta a la nativa. Este problema se puede intensificar en contextos educativos bilingües, donde los docentes deben adaptar el contenido y la lengua al alumnado con NEE sin formación específica para ello. Estos hallazgos demuestran que la formación del profesorado en atención a la diversidad depende de los currículos académicos establecidos para cada especialidad. Sin embargo, esta área de conocimiento debería ser obligatoria en cada especialización para garantizar una educación de calidad a cada estudiante.

Otro aspecto notable de este estudio está relacionado con la organización de la práctica docente. El profesorado reconoce reprogramar constantemente su programación para adaptarla a las necesidades de su alumnado. Este hecho puede resultar positivo si entendemos que observan regularmente a sus estudiantes; no obstante, también puede deberse a que su falta de formación en atención a la diversidad les haga probar nuevas fórmulas constantemente para intentar cubrir las necesidades de su alumnado. Esta idea puede tener relación con las respuestas de los docentes sobre el uso del libro de texto: la mayoría aseguró que tienden a diseñar actividades especiales para alumnado con NEE en lugar de usar material estándar.

Adicionalmente, el sexo del profesorado parece influir en la atención a la diversidad en la dimensión "Opinión sobre la atención a la diversidad", siendo los maestros menos conscientes de la importancia de esta. No obstante, ambos coinciden en su falta de formación y actúan de manera similar a la hora de programar su docencia o al aplicar diferentes metodologías para atender las necesidades de su alumnado. Sin embargo, estos aspectos arrojaron resultados diferentes cuando se cruzaron con las variables sociodemográficas "edad" y "experiencia laboral": los docentes más jóvenes son los que parecen mostrar más consciencia sobre la importancia de incluir la atención a la diversidad en sus programaciones docentes. Por el contrario, según nuestra muestra, los años de experiencia docente no influyen en la atención a la diversidad.

Finalmente, no existen diferencias significativas entre las concepciones y la gestión de la atención a la diversidad de los maestros y maestras de Educación Infantil y Primaria, de acuerdo con los resultados de nuestra muestra.

A pesar de los resultados obtenidos, este estudio presenta ciertas limitaciones que deberán ser resueltas en futuras investigaciones. En primer lugar, el tamaño de la 
muestra debe incrementarse para aumentar la representatividad. En segundo lugar, el cuestionario como instrumento de investigación socio-educativa debe complementarse con otros, como entrevistas y observación directa en el aula (Rodríguez Gómez y Valldeoriola Roquet, 2009). Finalmente, a pesar de que el cuestionario se diseñó intentando reducir el sesgo, el tema de este sondeo invita a contestar de manera ética en lugar de proporcionar opiniones reales. Por ello, es necesario ampliar la investigación introduciendo distintos instrumentos de recolección de datos y ampliando la muestra no solo en número de docentes, sino incluyendo también la opinión de los equipos directivos y, por supuesto, del alumnado, al igual que se pueden ampliar los contextos educativos por regiones, barrios, etc.

\section{Notas}

1. EI DUA puede definirse como el diseño de currículos accesibles para todo el alumanado que incluyan la diversidad de los estudiantes para evitar posibles barreras educativas derivadas de currículos inflexibles que no tienen en cuenta las situaciones personales o cognitivas de los alumnos (Díez y Sánchez, 2015).

La codociencia consiste en el trabajo compartido de dos docentes en la misma aula para permitir que los alumnos con necesidades educativas especiales y sin ellas trabajen a la vez y en el mismo espacio (Casserly y Padden, 2018).

2. El rango promedio es un estadístico necesario para interpretar los datos de la prueba $\mathrm{H}$ de Kruskal-Wallis. La existencia de valores muy dispares en dichos promedios puede considerarse un indicio de que la hipótesis alternativa es falsa.

\section{Referencias}

Achinstein, B y Athaneses, SZ (2005). Focusing new teachers on diversity and equity: Toward a knowledge base for mentors. Teaching and Teacher Education, 21(7), 843-862. https://doi.org/10.1016/j.tate.2005.05.017

Álvarez Duran, N (2009). Programar en educación especial. Innovación y experiencias educativas, 15, 1-8.

Araque, N y Barrio de la Puente, JL (2010). Atención a la diversidad y desarrollo de procesos educativos inclusivos. Prisma Social, 4, 1-37.

Arjona Fernández, ML (2010). Importancia y elementos de la programación didáctica. Hekademos: revista educativa digital, 7, 5-22.

Azorín, CM (2017). Análisis de instrumentos sobre educación inclusiva y atención a la diversidad. Revista Complutense de Educación, 28(4), 1043-1060. http://dx.doi.org/10.5209/RCED.51343

Bravo, LI (2013). Percepciones y opiniones hacia la educación inclusiva del profesorado y de los equipos directivos de los centros educativos de la Dirección Regional de Enseñanza de Cartago en Costa Rica. (Tesis doctoral, Universidad de Alicante). https://dialnet.unirioja.es/servlet/tesis?codigo $=55248$ 
Casserly, MA y Padden, A (2018). Teachers' views of co-teaching approaches in addressing pupils with special education needs (SEN) in multi-grade classrooms. European Journal of Special Needs Education, 33(4), 555-571. https://doi.org/10.1080/08856257.2017.1386315

Cejudo, J, Díaz, MV, Losada, L y Pérez-González, JC (2016). Necesidades de formación de maestros de infantil y primaria en atención a la diversidad. Bordón. Revista de pedagogía, 68(3), 23-39. https://doi.org/10.13042/Bordon.2016.68402

Díez, VE y Sánchez, FS (2015) Diseño universal para el aprendizaje como metodología docente para atender a la diversidad en la universidad. Aula abierta, 43(2), 8793. https://doi.org/10.1016/j.aula.2014.12.002

Echeíta, G (2005) Perspectivas y dimensiones en las políticas de atención a la diversidad. Alambique, Didáctica de las ciencias experimentales, 44, 7-16.

García Rubio, J (2017). Evolución legislativa de la educación inclusiva en España. Revista Nacional e Internacional de Educación Inclusiva, 10(1), 251-264.

García Vidal, J, González Manjon, D y Herrera Lara, JA (2005). Guía para la programación e intervención en Educación Especial. Madrid: EOS.

Hagiwara, M, Shogren, KA, Thompson, JR, Burke, KM, Uyanik, H, Amor, AM, Vergudo, MA y Aguayo, V (2019). International Trends in Inclusive Education Intervention Research: A Literature Review. Education and Training in Autism and Developmental Disabilities, 54(1), 3- 17.

Molina Olavarría, Y (2015). Necesidades educativas especiales, elementos para una propuesta de inclusión educativa a través de la investigación acción participativa. El caso de la Escuela de México. Estudios Pedagógicos, 41, 147-167. https://dx.doi.org/10.4067/S0718-07052015000300010

Montero, I y León, OG (2007). A guide for naming research studies in Psychology. International Journal of clinical and Health psychology, 7(3), 847-862.

Naciones Unidas (2006). Convención sobre los derechos de las personas con discapacidad.

https://www.un.org/esa/socdev/enable/rights/convtexts.htm\#convtext

Nogales Salamanqués, M (2012). La atención a la diversidad en la Educación Primaria: actitud y formación de los maestros (Tesis doctoral, Universidad Complutense de Madrid). https://fdocuments.ec/document/universidad-complutense-demadrid-facultad-de-naladiversidadenla-del.html

Rodríguez Gómez, D y Valldeoriola Roquet, J (2009). Metodología de la investigación. Cataluña: Universitat Oberta de Catalunya.

Roselló Ramón, MR (2010). El reto de planificar para la diversidad en la escuela. Revista Iberoamericana de Educación, 51(4), 1-10.

Sales Ciges, MA, Moliner García, MO y Sanchiz Ruiz, ML (2001). Actitudes hacia la atención a la diversidad en la formación inicial del profesorado. Revista Electrónica Interuniversitaria de Formación del Profesorado, 7(4), 1-7. 
Smyth, F, Shevlin, M, Buchner, T, Biewer, G, Flynn, P, Latimier, Šiška, J, Toboso-Martín, M, Rodríguez Díaz, S y Ferreira, M (2014). Inclusive education in progress: policy evolution in four European countries. European Journal of Special Needs Education, 29(4), 433-445. https://doi.org/10.1080/08856257.2014.922797

UNESCO (2005). Guidelines for inclusion: Ensuring Access to Education for All. France: ERIC

Clearinghouse. http://www.ibe.unesco.org/sites/default/files/Guidelines_for_Inclusion_UNESCO _2006.pdf

UNICEF (2006). Convención sobre los derechos del niño. España: Rex Media. https://www.un.org/es/events/childrenday/pdf/derechos.pdf. 
Anexo

\section{Cuestionario}

\section{Datos sociodemográficos}

1. Indique su sexo:

- Hombre

- Mujer

2. Indique el rango de edad al que pertenece

- De 20 a 30 años

- De 31 a 40 años

- De 41 a 50 años

- De 51 a 60 años

- Más de 60 años

3. Indique su titulación universitaria:

- Maestro/a de Educación Infantil

- Maestro/a de Educación Primaria

4. Especifique su especialidad si procede:

5. Indique, si las tuviese, otras titulaciones superiores después de haber obtenido su título universitario (cursos de especialización, máster o similar):

6. Indique el número de años de experiencia laboral docente:

\section{Formación recibida}

7. Indique su grado de acuerdo o desacuerdo en las siguientes afirmaciones

\begin{tabular}{|c|c|c|c|c|c|c|}
\hline & 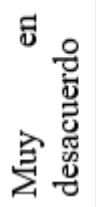 & 氠㟒 & : & 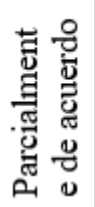 & 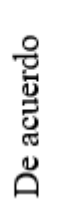 & 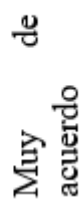 \\
\hline $\begin{array}{l}\text { La formación que recibí en la universidad acerca } \\
\text { de cómo trabajar con alumnos que presentan } \\
\text { necesidades educativas especiales fue suficiente. }\end{array}$ & & & & & & \\
\hline $\begin{array}{l}\text { El centro en el que trabajo me proporciona cursos } \\
\text { o seminarios para ampliar mi formación en } \\
\text { atención a la diversidad. }\end{array}$ & & & & & & \\
\hline $\begin{array}{l}\text { A lo largo de mi experiencia como docente, me he } \\
\text { encontrado con casos de alumnado con } \\
\text { necesidades educativas especiales que no he } \\
\text { sabido gestionar adecuadamente por falta de } \\
\text { formación. }\end{array}$ & & & & & & \\
\hline $\begin{array}{l}\text { Creo que si tuviese un mayor conocimiento sobre } \\
\text { necesidades educativas especiales podría atender } \\
\text { mucho mejor a la diversidad dentro del aula. }\end{array}$ & & & & & & \\
\hline $\begin{array}{l}\text { Considero que me hace falta formación en el } \\
\text { ámbito de la atención a la diversidad para cubrir } \\
\text { las necesidades de mis alumnos por completo. }\end{array}$ & & & & & & \\
\hline
\end{tabular}


Forma de programar

8. Indique con qué frecuencia realiza las siguientes actividades docentes:

\begin{tabular}{|c|c|c|c|c|c|c|}
\hline & 莺 & 总 & 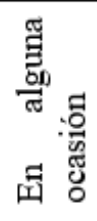 & 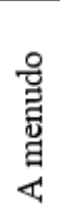 & 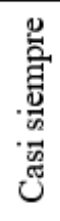 & 总 \\
\hline $\begin{array}{l}\text { Realizo la programación anual al principio de } \\
\text { curso. }\end{array}$ & & & & & & \\
\hline $\begin{array}{l}\text { Programo mi actividad docente al principio y de } \\
\text { cada unidad didáctica o tema nuevo. }\end{array}$ & & & & & & \\
\hline Programo mi actividad docente semanalmente. & & & & & & \\
\hline $\begin{array}{l}\text { Incluyo un apartado específico de atención a la } \\
\text { diversidad en mi programación anual. }\end{array}$ & & & & & & \\
\hline $\begin{array}{l}\text { Voy adaptando mi actividad docente según voy } \\
\text { observando las necesidades de mis alumnos. }\end{array}$ & & & & & & \\
\hline $\begin{array}{l}\text { Programo actividades específicas diarias para mis } \\
\text { alumnos de necesidades educativas especiales que } \\
\text { no pueden seguir el ritmo general de la clase. }\end{array}$ & & & & & & \\
\hline
\end{tabular}

\section{Metodologías}

9. Seleccione todas las opciones que representen su forma de actuar cuando tiene alumnos con necesidades educativas especiales en el aula

- Elaboro material extra para que trabajen dentro del aula.

- Me guío por libro de texto que corresponda a su nivel curricular.

- Intento programar para que el temario de los niños con desfase curricular y los niños que no presentan dificultades coincida en algún punto.

- Conozco estrategias educativas para incluir a los alumnos con necesidades educativas especiales en el ritmo normal de la clase. 
Opiniones sobre la atención a la diversidad

10. Indique su grado de acuerdo o desacuerdo con las siguientes afirmaciones:

\begin{tabular}{|c|c|c|c|c|c|c|}
\hline & 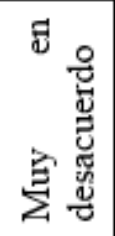 & 国 & 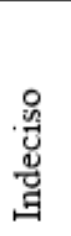 & 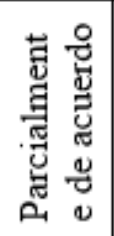 & 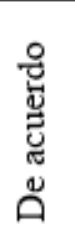 & 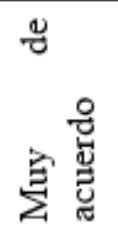 \\
\hline \multicolumn{7}{|l|}{$\begin{array}{l}\text { Trabajar la inclusión educativa en las aulas } \\
\text { favorece la integración social de alumnos con } \\
\text { discapacidad. }\end{array}$} \\
\hline \multicolumn{7}{|l|}{$\begin{array}{l}\text { Los maestros y maestras especialistas en } \\
\text { necesidades educativas junto con el equipo } \\
\text { directivo son los responsables de integrar a los } \\
\text { alumnos ACNEAE en centros ordinarios. }\end{array}$} \\
\hline \multicolumn{7}{|l|}{$\begin{array}{l}\text { Todo el equipo docente junto con el equipo } \\
\text { directivo son los responsables de integrar a los } \\
\text { alumnos ACNEAE en centros ordinarios. }\end{array}$} \\
\hline \multicolumn{7}{|l|}{$\begin{array}{l}\text { E1 alumnado con necesidades educativas } \\
\text { especiales debería ser atendido a tiempo completo } \\
\text { por maestros y maestras especializados. }\end{array}$} \\
\hline \multicolumn{7}{|l|}{$\begin{array}{l}\text { Las familias deben trabajar en conjunto con el } \\
\text { colegio para favorecer la inclusión del alumnado } \\
\text { con necesidades educativas especiales en el centro } \\
\text { escolar. }\end{array}$} \\
\hline $\begin{array}{l}\text { Los maestros y maestras deberían atender al } \\
\text { mismo tiempo y de igual manera las necesidades } \\
\text { de los alumnos con dificultades y las de los }\end{array}$ & & & & & & \\
\hline
\end{tabular}

